\title{
OPEN AND UNIFORMLY OPEN RELATIONS
}

\author{
BY P. MAH AND S. A. NAIMPALLY \\ Communicated May 6, 1974
}

\begin{abstract}
A general open mapping theorem is proved when the domain is an Efremovic proximity space. This is then used to prove several results on relations which are generalizations of results due to Kelley, Pettis and Weston. Applications to functional analysis are given.
\end{abstract}

1. Introduction. In a recent topology conference at Charlotte, Professor B. J. Pettis posed several problems concerning open and uniformly open relations. This paper is a brief announcement of the results of our investigation to answer some of these questions; details with proofs will appear elsewhere. Most of the terms used are well known and will be found in Kelley [3]. If $(X, \delta)$ is a proximity space, $Y$ a topological space and $R \subset X \times Y$ is a relation, then $R$ is weakly open iff for each $y \in Y, A \subset X, y \in R[A]^{-}$implies $R^{-1}[y] \delta A$ (Poljakov [7]). If $R$ is injective and open then $R$ is weakly open. Also if $(X, d)$ is a metric space (with the induced metric proximity), $Y$ is a Morita uniform space and $R$ is uniformly open, then $R$ is weakly open.

2. Main results.

2.1 THEOREM. If $(X, \delta)$ is an Efremovic proximity space, $Y$ a topological space, $R \subset X \times Y$ a weakly open and nearly open relation, then $R$ is open.

2.2 THEOREM. If $(X, d)$ is a metric space, $(Y, \Omega)$ a Morita uniform space, $R \subset X \times Y$ is weakly open and uniformly nearly open if and only if $R$ is uniformly open.

2.3 THEOREM. If $(X, \delta)$ is an Efremovix proximity space, $Y$ a topological space, $R \subset X \times Y$ an injective relation, then $R$ is open if and only if $R$ is weakly open and nearly open.

AMS (MOS) subject classifications (1970). Primary 54C10, 54C60, 46A30; Secondary $54 \mathrm{E} 05,54 \mathrm{E} 15$.

Key words and phrases. Open relations, nearly open, weakly open, Morita uniformity, proximity, uniformly open, uniformly nearly open, nearly continuous. 
3. Generalizations of Weston and Kelley theorems.

3.1 Theorem (CF. Weston [8, TheOREM 8]). Let $(X, d)$ be a complete metric space, $Y$ a Hausdorff space, $R \subset X \times Y$ a point-compact, USC nearly open bijective relation. Then $R$ is open.

3.2 Theorem (CF. Kelley [3, Theorem 6.36]). Let $(X, d)$ be a complete metric space, $(Y, \Omega)$ a Morita uniform space, and let $R \subset X \times Y$ have a closed graph. If $R$ is uniformly nearly open, then $R$ is uniformly open.

If in (3.1) we replace $Y$ by a $T_{4}$-space and $R$ by a point-closed relation, the result remains true. Theorem 3.1 and the above modification give analogous dual results concerning the implication "nearly continuous implies continuous". Theorem (3.2) yields an improvement of a result of Brown [1] to closed graphs.

4. Recent results of Pettis. Here we generalize recent results of Pettis [6].

4.1 Theorem. Suppose $Y$ is Hausdorff, $R \subset X \times Y$ is a bijective nearly open relation with a metrically complete graph. Then $R$ is open.

4.2 THEOREM. If $X$ and $Y$ are metrically complete, $R \subset X \times Y$ is a bijective nearly open relation with a closed graph, then $R$ is open.

Each of (4.1) and (4.2) includes two results of Pettis, one concerning open mappings and another concerning continuous mappings. We give a sample of the latter.

4.3 Theorem (Pettis [6]). If $X$ is Hausdorff and $f: X \rightarrow Y$ is a nearly continuous function with a metrically complete graph, then $f$ is continuous and $X$ is metrically complete.

\section{REFERENCES}

1. L. G. Brown, Note on the open mapping theorem, Pacific J. Math. 38 (1971), 25-28. MR 46 \#7434.

2. T. Husain, Introduction to topological groups, Saunders, Philadelphia, Pa., 1966. MR 34 \#278.

3. J. L. Kelley, General topology, Van Nostrand, Princeton, N. J., 1955. MR 16, 1136.

4. K. Morita, On the simple extension of a space with respect to a uniformity. I, Proc. Japan Acad. 27 (1951), 65-72. MR 14, 68.

5. S. A. Naimpally and B. D. Warrack, Proximity spaces, Cambridge Tracts in Math. and Math. Phys., no. 59, Cambridge Univ. Press, New York, 1970. MR 43 \#3992. 
6. B. J. Pettis, Some topological questions related to open mapping and closed graph theorems, Charlotte Topology Conference, March 1974 (unpublished).

7. V. Z. Poljakov, Open mappings of proximity spaces, Dokl. Akad. Nauk SSSR 155 (1964), 1014-1017 = Soviet Math. Dokl. 5 (1964), 545-548. MR 30 \#2460.

8. J. D. Weston, On the comparison of topologies, J. London Math. Soc. 32 (1957), 342-354. MR 20 \#1288.

DEPARTMENT OF MATHEMATICAL SCIENCES, LAKEHEAD UNIVERSITY, THUNDER BAY, ONTARIO, CANADA P7B 5E1 